\title{
Low-Power Agriculture IoT System with LoRa: Open Field Storage Observation
}

\author{
Esma Kökten* (Bahcesehir University, Istanbul, Turkey), \\ Bahadır Can Çalışkan (Bahcesehir University, Istanbul, Turkey), \\ Saeid Karamzadeh (Associate Professor, Bahcesehir University, Istanbul, Turkey), \\ Ece Gelal Soyak (Assistant Professor, Bahcesehir University, Istanbul, Turkey)
}

\begin{abstract}
The last decade has seen multiple research work on the use of LoRaWAN technology in smart agriculture. In open field storage, monitoring is crucial for increasing the logistics efficiency and improving crop quality. As battery maintenance is expensive in such areas, LoRa is a suitable technology that allows for lowpower communications. Within the framework of the research, a prototype has been built for tracking goods in open field storage. The battery lifetime has been analysed through calculations and measurements using LoRa communications. Our findings indicate that although sleeping current has the smallest percentage, it has the greatest effect on increasing the battery life, for longer battery life LoRa node must have a low self-discharge battery, and finally, sensors are the main battery depleting factor on the LoRa node.
\end{abstract}

Keywords - Agriculture; Battery lifetime; Food storage; IoT; LoRa; Low-power application.

\section{INTRODUCTION}

Low-Power Wide-Area Network (LPWAN) technologies have been recently used in various areas such as health, agriculture, animal tracking, smart buildings and industrial logistics [1]-[3]. LoRa, Sigfox and NB-IoT are popular examples of LPWAN. While these low-power technologies have drawbacks such as range, data size and data rate, with accurate definition of needs and good architecture, the disadvantages can be eliminated.

In agriculture, having long battery life is crucial because of the high replacement costs due to nodes being distributed over large land areas. Compared to alternative technologies (such as GSM, 3G and LTE), LPWAN technologies provide battery savings which ensure that nodes are always online and sustain relatively high output power. LPWAN technologies are preferable since power consumption should be minimised due to high maintenance effort in the replacement of batteries [4]. In particular, LoRa is preferred since it is simple to deploy, able to carry data over large distances, and operates in a license-free environment.

Nowadays, agriculture IoT systems are used in closed, protected environments such as greenhouses [5] and are generally focused on the production process. Different studies indicate that a large portion of food waste occurs in the storage process [6]. In terms of cost and energy, current solutions such as ventilation systems and coolers are not compatible with all kinds of food [7]. For instance, while aeration systems are one of the methods that can prolong the lifetime of food, it is not reasonable to introduce aeration systems or even physical security structures for poorer countries and cheaper crops [8]. Studies report that, due to cost, approximately $60-70 \%$ of food grains produced in poorer countries, such as India, are stored

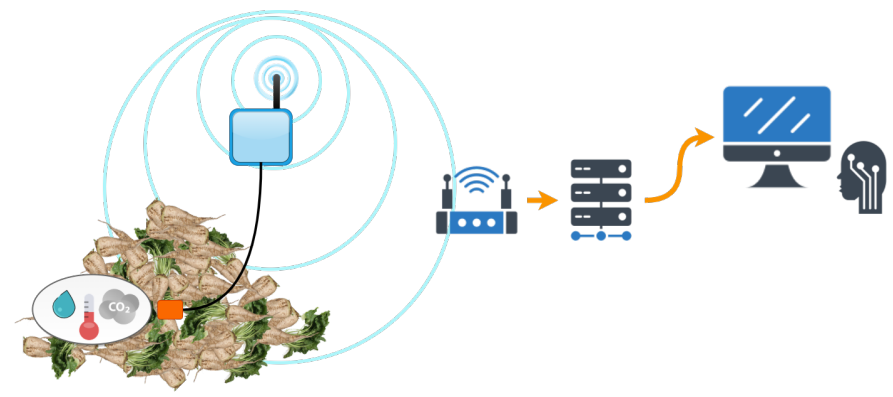

Fig. 1. Information flow from the sensor to the application server.

in indigenous storage structures [9]. It is therefore extremely necessary that the good stack is traced and analysed. By using scientific storage techniques, such losses can be reduced to as small as $2 \%$ [6]. Improving the management of open field food storage can be accomplished by constantly monitoring the stock and deriving the status of spoilage using the data analysis. In this study, battery life has been examined to maximise the power usage and transmitting distance using LoRaWAN technology with the intention of combating food waste in cheaper crops stored in open field storage such as sugar beet. For each sensor node, our approach includes a waterproof case and an indicator balloon so that it can be easily identified in an open area. Online network providers and application server providers that are compliant with many protocols for IoT communication are used. The Things Network, which is an open community for users of LoRa, is such a service. It provides a Graphical User Interface (GUI) for network server development. Moreover, some of the application server providers for LoRaWAN are Cayenne MyDevices, ChirpStack

\footnotetext{
* Corresponding author.

E-mail: esmakokten@gmail.com
} 
and OpenSensors [10]. CayenneMyDevices is used in our study. Figure 1 shows the solution and the information flow to the application server from the sensor node. Using LoRa, $\mathrm{CO}_{2}$, temperature and humidity values obtained from the food stack are transmitted and concentrated on the gateway and transferred to the servers. This paper is an extension of our initial study [11]. The present study includes both theoretical analysis and the measurement-base assessment of battery life as well as introduces the use of LoRa technology. Besides, recommendations are made to reduce energy consumption based on inferences drawn by evaluating the variables that have the most impact on battery life.

The remaining part of this paper is structured as follows. The use of IoT applications for smart agriculture is presented in Section II. The architecture used in the research is clarified in Section III. Section IV focuses on system power usage applying LoRaWAN technology, and Section V criticises the theoretical analysis and system measurements. Finally, Section VI draws conclusions.

\section{RELATED WORK}

The recent years have seen several research efforts towards smart agriculture, only a few of which study post-harvest losses. In this section, we briefly discuss previous models for smart agriculture using IoT and present the different types of battery that have been used in the prior art for smart agriculture.

In [12], the authors consider a smart irrigation system with machine learning which helps reduce food waste caused by improper irrigation during production. The authors used the LoRa network with TDM-based MAC protocol [12].

In [13], the authors propose a mobile LoRaWAN gateway system that can be used to increase the efficiency and precision of the operations in greenhouses. The technology presented includes Raspberry Pi 3 B + powered Mini LoRa Gateway from Heltec. It is operated by an external battery. Sensors monitor humidity and temperature, and send the collected data to the LoRaWAN gateway; the data are then processed and displayed by online platforms. This study does not focus on calculating lifetime in different scenarios or maximising the use life with a single charge.

In [14], the authors examine two use cases for smart agriculture. In one of the use cases, the authors place a sensor toolkit at a depth of $10 \mathrm{~cm}$ to $60 \mathrm{~cm}$ in order to analyse soil properties and measure the permeability of agricultural land. The analysis has been repeated using the different spreading factor $(\mathrm{SF})$ and output power $(\mathrm{dBm})$ values. It has been shown that the developed prototype is able to transmit reliably at a depth of up to $60 \mathrm{~cm}$, at a distance of $350 \mathrm{~m}$ from the gateway; this way, the whole region has been covered. The study shows that data transmission can be performed even if the spreading factor is reduced from SF8 to SF10 at lower measuring points and thus the transmission rate is lowered from 3125 bps to 980 bps. This study does not examine power consumption.

Due to its low power consumption, a majority of low-power IoT applications for agriculture employ the LoRa technology [13], [15], [16]. Apart from the power consumption of the LoRa module, other factors may also affect the power consumption in the overall system. Previous work on long-range communication shows that communication at a higher range than the projected distance is usually needed in order to compensate for obstacles affecting signal quality [14]. In these and similar cases, SF and output power should be adjusted correctly so that battery and range optimization can be performed. In addition, another dominant factor in power consumption is the sensor. There are a few factors for determining the power consumption of the sensor in the context of smart agriculture using LoRa [10].

There have also been prior efforts to study the effect of battery self-discharge. In [17], the authors show that selfdischarge increases with age and cycling. Their calculations reveal that, for the cells stored at $27{ }^{\circ} \mathrm{C}$, the average selfdischarge rate is $7.8111 \%$ in 10 years. In addition, it has also been shown that the Acceleration Factor (AF) delays the battery depletion.

In [18], the authors show that the reference storage time increases with $\mathrm{AF}$ at higher temperature; listed $\mathrm{AF}$ values for storage temperatures of $27^{\circ} \mathrm{C}, 40^{\circ} \mathrm{C}, 60^{\circ} \mathrm{C}$ and $70^{\circ} \mathrm{C}$ have been shown to take values of $1.0,2.7,12.5$ and 26.8 , respectively [18].

Given the existing studies described above, it is possible to summarise the novelty values of the present research. To the best of our knowledge, it is the first study that evaluates battery life both using battery calculations and measurements. Besides, unlike the existing research on smart agriculture with IoT, in this paper post-harvest storage with IoT is examined. Finally, selfdischarge results are compared with calculations and measurements in various types of batteries.

\section{PhySICAL ARCHITECTURE}

\section{A. Components of the LoRa Network}

In the system studied in this paper, the LoRa network consists of four main parts: a sensor node, a LoRa gateway, a network server and an application server.

At the sensor end, the microcontroller unit (MCU) reads the sensor value via $I^{2} C$ and sends the data via Serial Peripheral Interface (SPI) to the LoRa module.

LoRa transceiver takes data from the SPI and packages it into frames, which are then sent to the gateway. This flow can be seen in Fig. 2. Next, the data are received by the LoRa transceiver that is on the gateway. A Raspberry Pi 3 is used to relay the packets to the Internet over $802.11 \mathrm{n} 2.4 \mathrm{GHz} \mathrm{WiFi}$. The gateway is shown in Fig. 3.

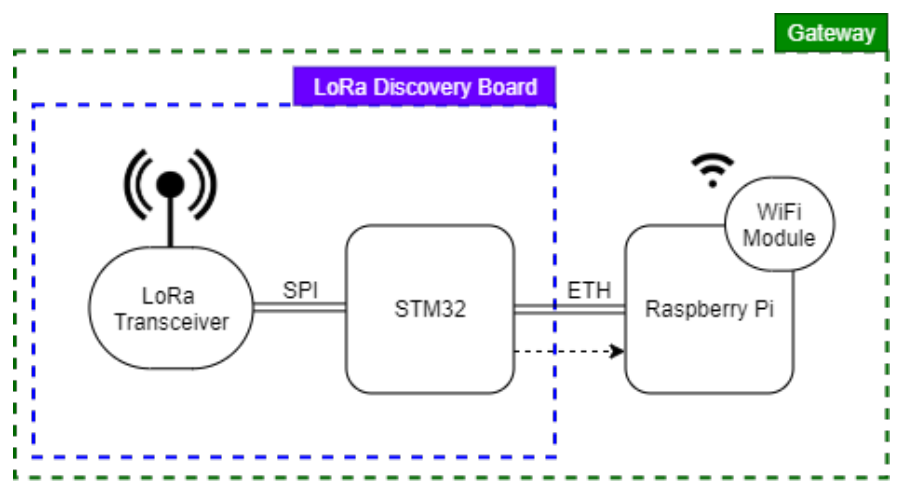


Fig. 2. Sensor node diagram.

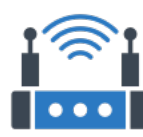

Nucleo Gateway

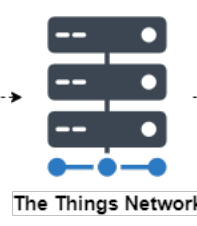

Fig. 3. Gateway diagram.
The Things Network server handles the incoming packets from the gateway and forwards them to related application servers (Fig. 4). Cayyenne MyDevices application server stores the collected data and presents them on its Graphical User Interface (GUI).

\section{B. LoRaWAN Evaluation Boards}

B-L072Z-LRWAN1 is used as a sensor node and it is powered by the CMWX1ZZABZ Murata module consisting of STM32L072CZ microcontroller and SX1276 transceiver. The B-L072Z-LRWAN1 discovery kit includes an ST-LINK/V2-1 embedded debug tool, an antenna, Arduino Uno V3 connectors and a USB OTG connector in Micro-B format. The transceiver features the LoRa protocol, operating in the $860-930 \mathrm{MHz}$ frequency range.

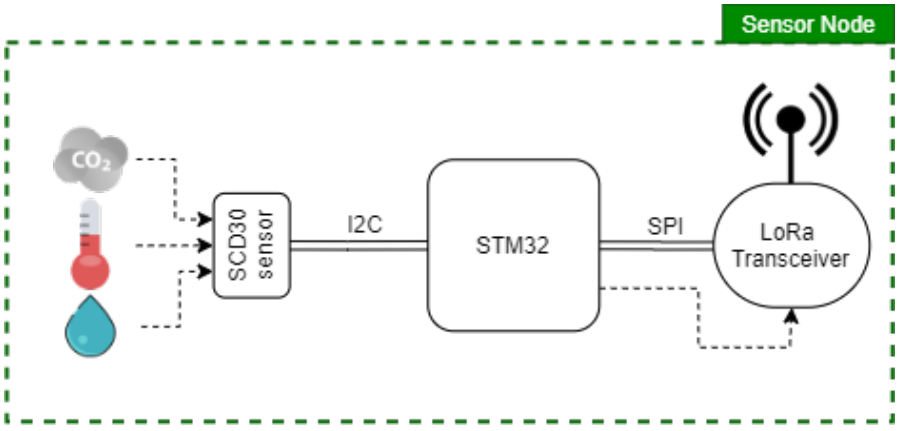

Fig. 4. Network Architecture.

The transceiver supports $+14 \mathrm{dBm}$ and $+20 \mathrm{dBm}$ selectable output power levels. NUCLEO-F746ZG is used as a gateway and it is based on a high-performance STM32F7-series ARM 32-bit microcontroller. ARM Cortex-M7 has an MCU operating at $216 \mathrm{MHz}$ with $1 \mathrm{MB}$ Flash memory and $320 \mathrm{kB}$ SRAM. It has RisingHF ARDUINO expansion board (LRWAN GS HF1) that controls the LoRaWAN communication. It is compliant with the IEEE-802.3-2002 Ethernet specification.

\section{Printed Circuit Board (PCB)}

A PCB has been designed to organise the LoRaWAN evaluation board pins. This extension board contains a charging circuit, a switch circuit, connectors, an indicator LED, and a button for controlling the power on the board. MCP73831 is used to control battery charging; it provides $500 \mathrm{~mA}$ current. The battery is connected to the circuit via a jumper cap so th a it can be switched between charging and consuming.

\section{Switching Circuit}

The directly connected sensor remains open all the time. To fix that issue, a switch circuit is designed and integrated into the PCB. It has been observed that controlling the sensor through a switch reduces $19 \mathrm{~mA}$ of current. The switch circuit contains two active components: 2n2222 as BJT and IRF9530 as p-type MOSFET. The switching circuit yields $3.6 \mathrm{~V}$ output when $3.3 \mathrm{~V}$ is supplied from the digital output of the micro- controller GPIO. It allows us to control the sensor over the digital output of the microcontroller.

\section{E. Raspberry Pi 3}

It is a computer board powered by a 64-bit $1.2 \mathrm{GHz}$ Broadcom BCM2837 CPU and 1 GB RAM. Raspberry Pi 3 has BCM43438 wireless LAN and Bluetooth Low Energy (BLE) network cards on board. The Raspberry Pi board is responsible for transmitting network packets received from the gateway on the eth0 interface to the Internet via WiFi.

\section{F. Sensor Components}

SCD30 is a Sensirion-manufactured portable carbon dioxide, temperature, and humidity sensor (Fig. 5). It is completely calibrated during manufacturing; however, depending on the atmospheric pressure it is also possible to set a custom compensation. The sensor can itself communicate with a master device via the $I^{2} C$ or the UART bus. Repeatability in the $\mathrm{CO}_{2}$ measurement is $10 \mathrm{ppm}$ and the sensor consumes $19 \mathrm{~mA}$ average current at the measurement interval of $2 \mathrm{~s}$.

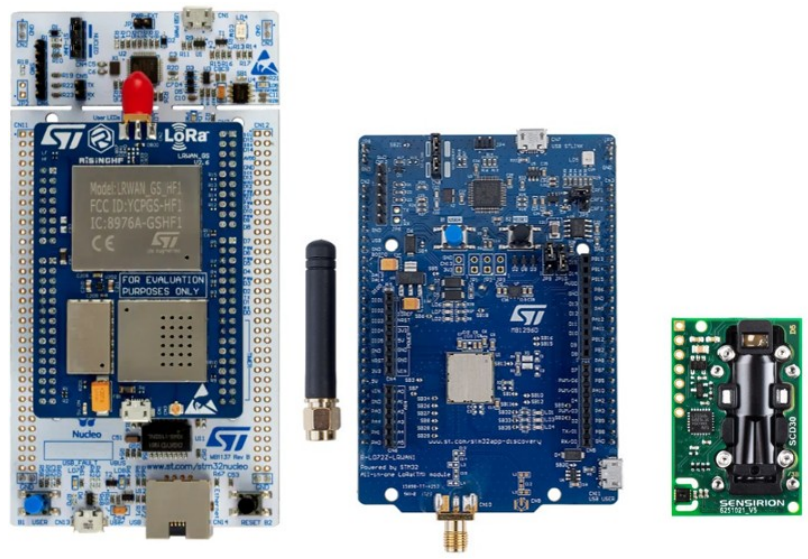

Fig. 5. NUCLEO board, B-L072-LRWAN1 and SCD30.

\section{BATTERY LIFE}

There are several variables influencing the battery life of the sensor node, such as sleep time, sensor, radio settings, temperature, as well as only battery-related variables, such as usable energy, form, health and self-discharge. The subsequent sub-sections illustrate two methods for computing power usage in LoRa sensor nodes with self-discharging batteries.

\section{A.Power Consumption}

The present study primarily focuses on the best and worstcase battery life values using SF7 and SF12 with the output power of $14 \mathrm{dBm}$ and $20 \mathrm{dBm}$. Calculations are made according to the sensor node that transmits 20 bytes of data in each transmission. Sensor nodes repeat the same cycle until the battery becomes depleted. One cycle of the device is determined based on the voltage measured by an oscilloscope over a $1 \Omega$ resistor that is serial to the entire circuit, as shown in Fig. 6 . 
Voltage values can be converted into current values using Ohm's Law. This approach is more accurate than using datasheet values for calculating the battery life of the end product. However, in this study the measured values are high due to the unnecessary elements on the evaluation board such as LEDs, debugger and regulator. To eliminate this effect, current values of the microcontroller and LoRa module are measured and used together with the values taken from the datasheets.

TABLE I

Current and Duration Values of the Given Mode

\begin{tabular}{|c|c|c|c|}
\hline \multirow{2}{*}{ Operation } & \multicolumn{3}{|c|}{ Components } \\
\cline { 2 - 4 } Sleep & $\begin{array}{c}\text { UTMit } \\
\text { SX1276 SCD30 }\end{array}$ & $\begin{array}{c}37 \mu \mathrm{A} \\
\text { Off Off }\end{array}$ & $1 \mathrm{~h}$ \\
\hline \multirow{2}{*}{ Measure } & $\begin{array}{c}\text { STM32L072CZ } \\
\text { SCD30 }\end{array}$ & $\begin{array}{c}140 \mu \mathrm{A} \\
19 \mathrm{~mA}\end{array}$ & $72 \mathrm{~s}$ (heat up) $\approx 80 \mathrm{~s}$ \\
\hline Send & CMWX1ZZABZ & $\begin{array}{c}47 \mathrm{~mA}(\mathrm{SF} 7) \\
128 \mathrm{~mA}(\mathrm{SF} 12)\end{array}$ & $\begin{array}{c}56.58 \mathrm{~ms}(\mathrm{SF} 7)^{3} \\
1318.91 \mathrm{~ms}(\mathrm{SF} 12)\end{array}$ \\
\hline \multirow{2}{*}{ Receive } & CMWX1ZZABZ & $21.5 \mathrm{~mA}$ & $\begin{array}{c}10.2 \mathrm{~ms}(\mathrm{SF} 7) \\
328 \mathrm{~ms}(\mathrm{SF} 12)\end{array}$ \\
\hline
\end{tabular}

Table I shows the current and duration values with respect to the datasheets of CMWX1ZZABZ (MURATA module consists of STM32L072CZ+SX1276) and SCD30 (Sensirionmanufactured compact carbon dioxide, temperature and humidity sensor). These measurements have been carried out using an Owon XDS3202 Oscilloscope and an Owon OW18B Multimeter.

Table II shows the constant current values of the board sleeping phase and external circuitry measured with the multimeter mentioned above. Available battery capacity divided by the power consumed the system in one cycle gives the maximum number of cycles that the device can continue on running.

TABLE II

MEASURED VALUES

\begin{tabular}{|c|c|c|}
\hline Unit & State & Measured Current \\
\hline $\begin{array}{c}\text { STM32L072CZ } \\
\text { SX1276 }\end{array}$ & Sleep & $\begin{array}{c}30.6 \mu \mathrm{A} \\
<1 \mu \mathrm{A}\end{array}$ \\
\hline Circuitry $^{1}$ & - & $38.2 \mu \mathrm{A}$ \\
\hline
\end{tabular}

Equation (1) can be used to find battery lifetime after calculating the duration of one cycle. The battery life $T_{\text {bat }}$ is

$$
\begin{gathered}
T_{\text {bat }}=N \cdot T_{\text {cycle }}, \\
N=\frac{E_{\text {bat }}}{E_{\text {cycle }}},
\end{gathered}
$$

where $T_{\text {cycle }}$ is the time passed in one cycle, $N$ is maximum number of cycle that battery lasts, $E_{\text {bat }}$ is the capacity of the battery and $E_{\text {cycle }}$ is the electric charge consumed in one cycle.

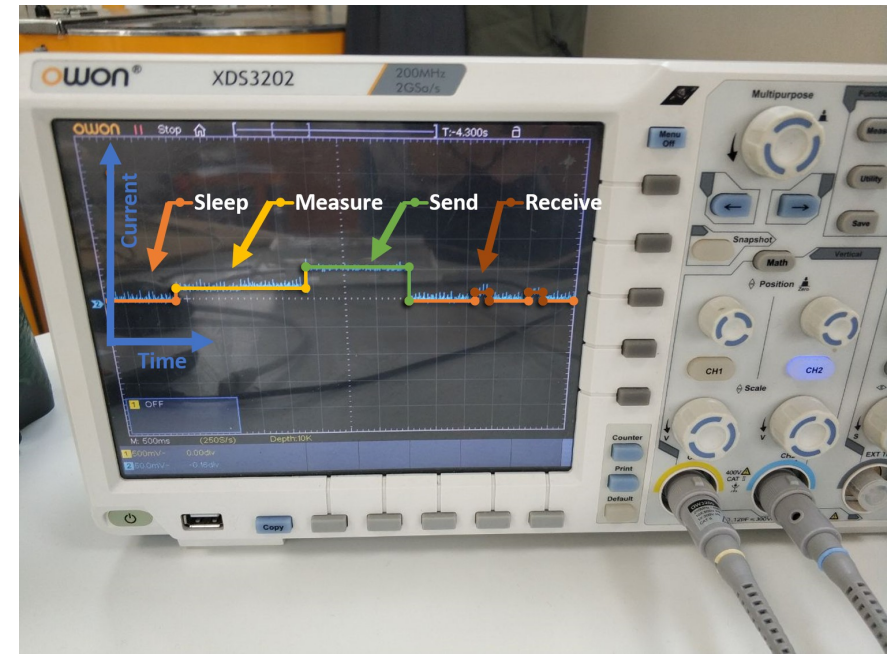

Fig. 6. One cycle in oscilloscope screen.

Power consumption of one cycle can also be interpreted as the area under the graph in Fig. 6. Therefore, which operation affects the battery lifetime more is not only related to the current values it takes, but also to the duration of the operation. Further explanation of power consumption is provided in Section V.

\section{B. Self-Discharge}

Self-discharge has a great weight in low-power applications. In batteries, a certain degree of self-discharge may be expected depending on the system electrochemistry, the purity of reagents and the temperature when batteries are charged and left on an open circuit. What is called the shelf-life of batteries is determined by the rate of self-discharge, which typically decreases over time.

In this study, a rechargeable Li-Ion battery has been preferred as it is more cost-effective and maximum current limit of $\mathrm{Li} / \mathrm{SOCI}_{2}$ battery is insufficient for the use case. The selfdischarge of Li-ion is relatively steady during its service life under normal conditions; however, full state-of-charge and elevated temperature cause a rise. (Self-discharge of all battery chemistries increases at higher temperatures, and the intensity normally doubles every $10^{\circ} \mathrm{C}$ [19].) Rechargeable Li-ion batteries have a self-discharge lower than $5 \%$ per month [20]. Another reason to choose a Li-ion battery instead of $\mathrm{Li} / \mathrm{SOCI}_{2}$ is that the rechargeable battery can be used to store power produced by renewable energy sources [21].

TABLE III

SELF-DISCHARGE OF LI-ION AND LISOCI $\mathrm{L}_{2}$ BATTERIES

\begin{tabular}{|c|c|c|c|c|c|c|c|}
\hline Battery & \multirow{2}{*}{ SOC } & $\mathbf{0}^{\circ} \mathbf{C}$ & $\mathbf{2 5}^{\circ} \mathbf{C}$ & $\mathbf{2 7}^{\circ} \mathbf{C}$ & $\mathbf{4 0}^{\circ} \mathbf{C}$ & $\mathbf{6 0}{ }^{\circ} \mathbf{C}$ & $\mathbf{7 0}^{\circ} \mathbf{C}$ \\
\cline { 1 - 7 } LiSOCI & \multirow{2}{*}{ Full } & - & - & $0.07 \%$ & $0.2 \%$ & $0.9 \%$ & $2 \%$ \\
\cline { 1 - 7 } & \multirow{2}{*}{ Li-Ion[19] } & $6 \%$ & $20 \%$ & - & - & $35 \%$ & - \\
\cline { 2 - 8 } & $40-60 \%$ & $2 \%$ & $4 \%$ & - & - & $15 \%$ & - \\
\hline
\end{tabular}

Non-rechargeable $\mathrm{Li} / \mathrm{SOCI}_{2}$ batteries have been mostly preferred in many prior LoRa research efforts due to their low self-discharging characteristics [22]. In Table III, the selfdischarge characteristics of the Li-ion battery have been compared to the characteristics of $\mathrm{Li} / \mathrm{SOCI}_{2}$ (lithium/thionyl chloride) battery as a reference model, as it has very low self- 
discharge characteristics (studies show that it has nearly one percent self-discharge [23]). In [18], the acceleration factors of the self-discharge at different temperatures have been revealed. The calculations of $\mathrm{Li} / \mathrm{SOCI}_{2}$ are made using the information provided by these authors.

\section{RESULTS AND DISCUSSION}

Figure 7 illustrates the power consumption of each phase for different LoRa configurations. The measurement phase consumes much greater energy compared to the other phases, due to both the time for the $\mathrm{CO}_{2}$ sensor to heat up and the high electric current it consumes. It may be concluded that there is a need for low-power compatible sensors for the $\mathrm{CO}_{2}$ measurements. In this section, sleep, measurement, send and receive features in a device cycle are verified with an oscilloscope. In addition, we individually analyse the effect of each operating mode on the battery life.

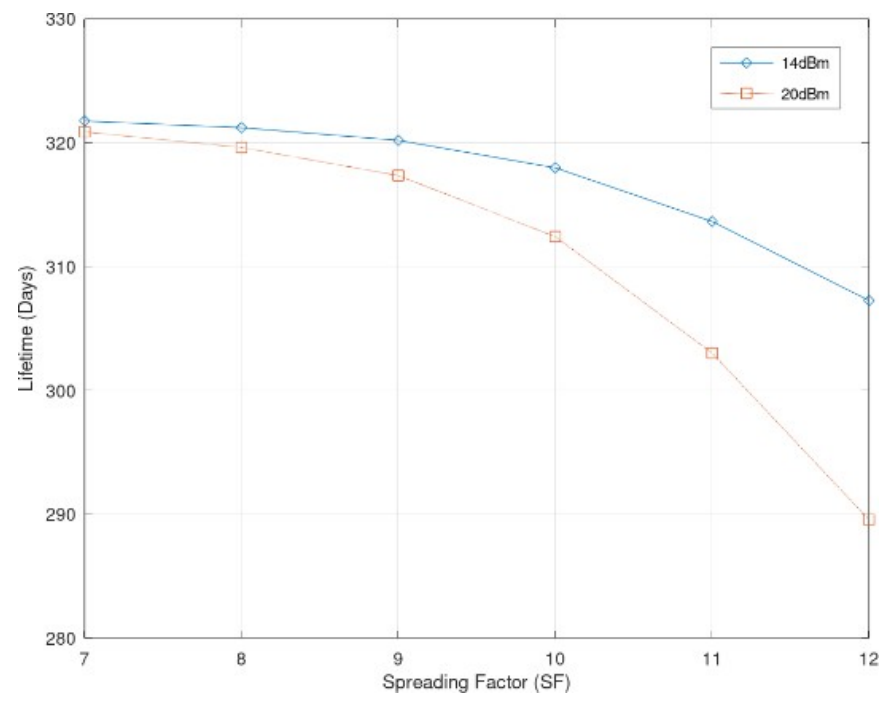

Fig. 7. Power consumption by the operations individually.

During the battery calculations presented earlier, the impact of reducing the current consumption of each operation mode by $1 \%$ on the battery life was calculated. Effect of $1 \%$ current improvement during the sleeping phase is $80 \%$, during the measurement phase it is $20 \%$, during the sending and receiving phase it is close to $0 \%$. It implies that $1 \%$ improvement on the sleeping current is equivalent to $4 \%$ improvement on the measured current. Thus, although battery optimisation for LoRa configurations such as SF and output power is a popular subject, it has a smaller impact on the big picture for this sensor node.

\section{A. Effects of LoRa Settings}

Battery life calculation is made for four different configurations with SF and output power for $3500 \mathrm{mAh} \mathrm{Li-ion}$ battery capacity. As can be perceived from Fig. 8, output power has a greater negative impact in SF12 than in SF7 because of the consequent increase in transmission time.

\section{B. Effects of the Sleep}

In non-real-time applications such as those described in this paper, sleeping time highly affects the lifetime. For the sensors used in such applications, sleep frequency may differ based on the food being monitored post-harvest. As discussed earlier, the sleeping current is one of the most effective consumers of the battery. Measuring battery voltage, using regulator, LEDs always open sensors to increase the draw.

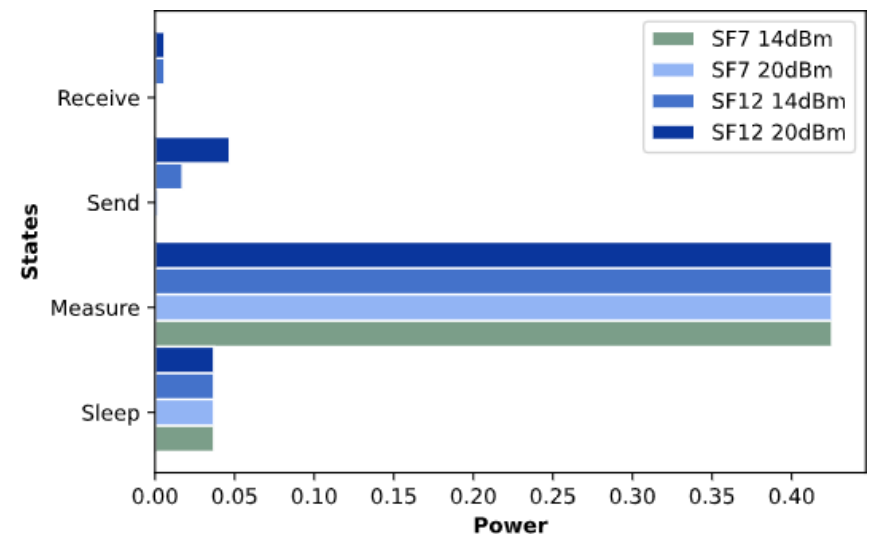

Fig. 8. Battery life graph.

To improve the current during sleep, a switching circuit is used for the sensor. Comparing 2 hours of sleep interval with the 1-hour case, the percentage of its role in power consumption is increased from $80 \%$ to $89 \%$.

\section{Effects of Self-Discharge}

While the previously calculated values are derived by using a constant battery capacity ( $3500 \mathrm{mAh})$, battery self-discharge is another factor that extremely affects the battery life. In our system, a monthly $5 \%$ of self-discharge causes $34 \%$ of drop in the battery life. Therefore, batteries are not compatible with this application. For low-power sensor node applications, low selfdischarge $\mathrm{LiFePO}_{4}, \mathrm{Li} / \mathrm{SOCI}_{2}$ and alkaline batteries can be preferred.

\section{CONCLUSION}

In this study, we have examined the battery lifetime using LoRaWAN technology for monitoring the storage process for open storage water-intensive goods such as sugar beets. Recommendations for reducing power use are identified by assessing variables that have a greater impact on the battery life. While the sleeping current is the smallest, it has the most important function to play in increasing the battery life. When developing a low-power sensor node, it is important to choose a low-power sensor and use a switching circuit to minimise the sleeping current. Another important aspect is choosing a battery that has low self-discharge. Further, to make efficient and affordable sensor nodes, sensor costs (both battery and price) should be decreased and new studies can be conducted on how to use sensors in the most battery efficient way. The key concept is to minimise post-harvest storage losses by designing a tool for improved monitoring and condition control of the goods. While it is a very basic method to estimate the battery life for the sensor nodes, there are other important factors affecting battery discharge such as elevated temperature, age, and state 
of charge. There is no feasible, easy to implement formula for calculating battery life based on these parameters. Furthermore, battery consumption can be observed using measurements made with more sophisticated equipment and a test setup can be prepared to validate the calculations.

\section{REFERENCES}

[1] W. Ayoub, A. E. Samhat, F. Nouvel, M. Mroue, and J.-C. Prévotet, "Internet of mobile things: Overview of lorawan, DASH7, and NB-IoT in LPWANs standards and supported mobility," IEEE Communications Surveys \& Tutorials, vol. 21, no. 2, pp. 1561-1581, 2018. https://doi.org/10.1109/COMST.2018.2877382

[2] Lora-Alliance, "Why lorawan is the logical choice for asset-tracking connectivity," White paper, April 2020.

[3] Semtech, "Lora devices: Smart agriculture real world solutions," White paper, 2021.

[4] F. Javed, M. K. Afzal, M. Sharif, and B.-S. Kim, "Internet of things (IoT) operating systems support, networking technologies, applications, and challenges: A comparative review," IEEE Communications Surveys \& Tutorials, vol. 20, no. 3, pp. 2062-2100, 2018 https://doi.org/10.1109/COMST.2018.2817685

[5] I. Mohanraj, K. Ashokumar, and J. Naren, "Field monitoring and automation using iot in agriculture domain," Procedia Computer Science, vol. 93, pp. 931-939, 2016. https://doi.org/10.1016/j.procs.2016.07.275

[6] D. Kumar and P. Kalita, "Reducing postharvest losses during storage of grain crops to strengthen food security in developing countries," Foods, vol. 6, no. 1, p. 8, 2017. https://doi.org/10.3390/foods6010008

[7] D. de Carvalho Lopes, J. H. Martins, A. F. Lacerda Filho, E. de Cas- tro Melo, P. M. de Barros Monteiro, and D. M. de Queiroz, "Aeration strategy for controlling grain storage based on simulation and on real data acquisition," Computers and Electronics in Agriculture, vol. 63, no. 2, pp. 140-146, 2008. https://doi.org/10.1016/j.compag.2008.02.002

[8] D. Grover and J. Singh, "Post-harvest losses in wheat crop in Punjab: Past and present," Agricultural Economics Research Review, vol. 26, no. 2, pp. 293-297, 2013.

[9] M. Sharon, C. Abirami, and K. Alagusundaram, "Grain storage management in india," Journal of Postharvest Technology, vol. 2, no. 1, pp. $12-24,2014$

[10] T. Bouguera, J.-F. Diouris, J.-J. Chaillout, and G. Andrieux, "Energy consumption modeling for communicating sensors using LoRa technology," in 2018 IEEE Conference on Antenna Measurements \& Applications (CAMA), Vasteras, pp. 1-4, 2018. https://doi.org/10.1109/CAMA.2018.8530593

[11] E. Kokten, B. C. Caliskan, S. Karamzadeh, and E. Gelal Soyak, "LowPowered agriculture IoT systems with LoRa," in 2020 IEEE Workshop on Microwave Theory and Techniques in Wireless Communications, Riga, Latvia, Sep. 2020. https://doi.org/10.1109/MTTW51045.2020.9244927

[12] Y.-C. Chang, T.-W. Huang, and N.-F. Huang, "A machine learning based smart irrigation system with LoRa P2P networks," in 2019 20th AsiaPacific Network Operations and Management Symposium (APNOMS). IEEE, 2019, pp. 1-4. https://doi.org/10.23919/APNOMS.2019.8893034

[13] S. Gutiérrez, I. Martínez, J. Varona, M. Cardona, and R. Espinosa, "Smart mobile LoRa agriculture system based on internet of things," in 2019 IEEE 39th Central America and Panama Convention (CONCAPAN XXXIX). IEEE, 2019, pp. 1-6. https://doi.org/10.1109/CONCAPANXXXIX47272.2019.8977109

[14] A. Grunwald, M. Schaarschmidt, and C. Westerkamp, "LoRaWAN in a rural context: Use cases and opportunities for agricultural businesses," in Mobile Communication Technologies and Applications; 24 ITGSymposium, VDE, 2019, pp. 1-6.

[15] J. D. Adriano, Y. C. Mendes, G. A. Marcondes, V. Furtado, and J. J. Rodrigues, "An IoT sensor mote for precision agriculture with several mac layer protocols support," in 2018 International Conference on Information and Communication Technology Convergence (ICTC). IEEE, 2018, pp. 684-688.

[16] W. R. Da Silva, L. Oliveira, N. Kumar, R. A. Rabêlo, C. N. Marins, and J. J. Rodrigues, "An internet of things tracking system approach based on LoRa protocol," in 2018 IEEE Global Communications Conference (GLOBECOM), IEEE, 2018, pp. 1-7.

https://doi.org/10.1109/GLOCOM.2018.8647984
[17] I. Buchmann, Batteries in a Portable World: A Handbook on Rechargeable Batteries for Non-engineers. Cadex Electronics Incorporated, 2016. [Online]. Available: https://books.google.com.tr/books?id=Nt2RnQAACAAJ

[18] S. Cheng, B. Li, Z. Yuan, F. Zhang, and J. Liu, "Development of a lifetime prediction model for lithium thionyl chloride batteries based on an accelerated degradation test," Microelectronics Reliability, vol. 65, pp. 274-279, 2016. https://doi.org/10.1016/j.microrel.2016.07.152

[19] I. Buchmann, Batteries in a portable world: a handbook on rechargeable batteries for non-engineers, 2nd ed. Cadex Electronics Inc, 2001.

[20] R. de Fazio, D. Cafagna, G. Marcuccio, and P. Visconti, "Limitations and characterization of energy storage devices for harvesting applications," Energies, vol. 13, no. 4, p. 783, 2020. https://doi.org/10.3390/en13040783

[21] F. Pitu and N. C. Gaitan, "Surveillance of SigFox technology integrated with environmental monitoring," in 2020 International Conference on Development and Application Systems (DAS), IEEE, 2020, pp. 69-72. https://doi.org/10.1109/DAS49615.2020.9108957

[22] N. Naik, "LPWAN technologies for IoT systems: choice between ultra narrow band and spread spectrum," in 2018 IEEE International Systems Engineering Symposium (ISSE), IEEE, 2018, pp. 1-8. https://doi.org/10.1109/SysEng.2018.8544414

[23] S. Jacob, "Don't overdesign your battery for IIOT applications," Tadiran Batteries, White paper, 2019.

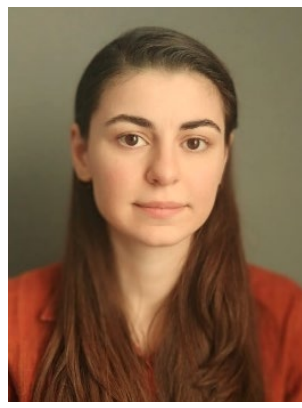

Esma Kökten received the B. sc. degree in Electrical \& Electronics Engineering and Computer Engineering from Bahcesehir University, Istanbul, Turkey, in 2020 and 2021 respectively. She has been working in automation industry as software developer since 2019. The area of scientific interest is Internet of Things. E-mail: esmakokten@gmail.com

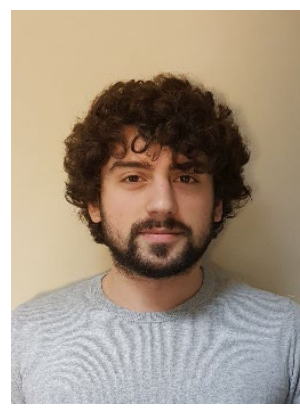

Bahadır Can Çalışkan received the B. sc. degree in Electrical \& Electronics Engineering from Bahcesehir University, Istanbul, Turkey, in 2020. $\mathrm{He}$ has been working in the energy industry as a hardware designer since 2019. The area of scientific interest is Internet of Things, electric vehicle charging stations and their impact on grid. E-mail: bahadrcan@gmail.com ORCID iD: https://orcid.org/0000-0002-8414$\underline{265 X}$

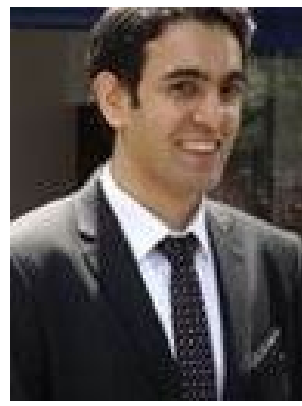

Saeid Karamzadeh received his MS and $\mathrm{Ph}$. D. degree in the Department of Communication Systems, Satellite Communication \& Remote Sensing program at Istanbul Technical University in 2013 and 2015, respectively. He won the award for the most successful Ph. D. thesis from Istanbul Technical University. He is an Assoc. Prof. Dr at Bahçeşehir University, Department of Electrical and Electronics Engineering currently. His research interests include remote sensing, radar, microwave, and antenna design. E-mail: saeid.karamzadeh@eng.bau.edu.tr ORCID iD: https://orcid.org/0000-0003-0669-0746 


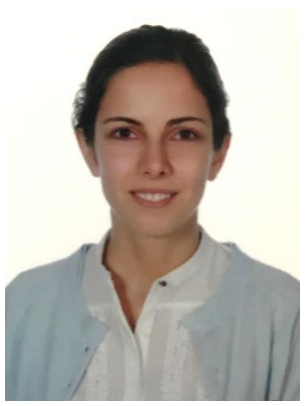

Ece Gelal Soyak received the B. sc. degree in Telecommunications Engineering from Sabanci University, Istanbul, Turkey, in 2004, and the $\mathrm{Ph}$. D. degree in Computer Science and Engineering from the University of California, Riverside, in 2009.From 2010 to 2018 she worked in the industry as a senior engineer, doing research, software development, and team leadership. In 2018 she joined the Computer Engineering department in Bahcesehir University, Istanbul, Turkey. Her research interests are in the field of wireless networks, with emphasis on the scalability and security of the Internet of Things, and use of machine learning for solving wireless networking problems.

E-mail: ece.gelalsoyak@eng.bau.edu.tr

ORCID iD: https://orcid.org/0000-0003-2410-6267 